\title{
Strength of Natural Soil Flocs
}

Motoyoshi Kobayashi ${ }^{1,2}$

${ }^{1}$ Laboratory of Water Environmental Engineering

Department of Biological and Environmental Engineering

University of Tokyo

Yayoi 1-1-1, Bunkyo-ku, Tokyo 113-8657

Japan

and

${ }^{2}$ Laboratory of Colloid and Surface Chemistry

Department of Inorganic, Analytical and Applied Chemistry

University of Geneva

Sciences II, 30 quai Ernest Ansermet, CH-1211 Geneva 4

Switzerland

${ }^{2}$ Present Address

Tel: +41-22-379 6055, Fax: +41-22-379 6069

Email: Motoyoshi.Kobayashi@unige.ch 


\section{Abstract}

To obtain the strength of flocs against breakup is crucial for controlling flocculation in water treatment and predicting transport of colloidal particles in aqueous environments. Recently, the author reported a method to obtain floc strength from a simple experiment of floc breakup subjected to a laminar converging flow. In this study, this method was applied to natural soil flocs. The flocs were formed by coagulation with $0.5 \mathrm{M} \mathrm{NaCl}\left(\mathrm{pH}\right.$ 5.4-5.5, $\mathrm{pH}$ 6.6) solutions, $0.1 \mathrm{M} \mathrm{CaCl}_{2}(\mathrm{pH}$ 6.4-6.9) solutions, or acidified distilled water with dilute $\mathrm{HCl}$ ( $\mathrm{pH}$ 5.6). Obtained floc strengths were $0.3 \mathrm{nN}, 0.7 \mathrm{nN}$ and $4 \mathrm{nN}$ for Na-coagulated flocs, Ca-coagulated flocs, and H-coagulated flocs, respectively. Also, floc strength did not change with floc size. These values of floc strengths were 1-3 orders smaller than those of flocs formed with polymer flocculants and/or precipitated ferric or aluminum coagulants.

Key Words: breakup, aggregate, converging flow, clay, Kunigami maaji 


\section{Nomenclature}

$\mathrm{A}_{\mathrm{c}, \max } \quad$ maximum elongation rate of flow along the centreline of flow into orifice given

by Eq.(3), (1/s)

Coefficient in Eq. (2), (-)

$\mathrm{d}_{\text {maj }}, \mathrm{d}_{\min } \quad$ Lengths of major and minor axes of the best-fit ellipse corresponding to

respective flocs $(\mathrm{m})$

F floc $\quad$ floc strength $(\mathrm{N})$

Fyd $\quad$ hydrodynamic force acting on floc (N)

Q volumetric rate of flow $\left(\mathrm{m}^{3} / \mathrm{s}\right)$

$\mathrm{R} \quad$ radius of orifice (m)

S $\quad$ surface area of ellipsoid $\left(\mathrm{m}^{2}\right)$

Greek letter

$\mu$

viscosity of fluid (Pas) 


\section{Introduction}

Colloidal particles in water environments tend to form flocs (aggregates of colloidal particles). Transport properties of particulates depend strongly on their size. Thus, to predict the transport of colloidal particles and contaminants sorbed on particles in aqueous environments (lakes, ocean, and groundwater), the prediction of size of flocs is desired (Droppo, 2001; Edzwald et al., 1974; Kretzschmar et al., 1999). It is considered that flocs are disrupted and then change their size when the hydrodynamic force exerted on flocs, $F_{\text {hyd }}$, exceeds the strength of flocs, $F_{\text {floc }}$ (Boller and Blaser, 1998; Kobayashi et al., 1999; Tambo et al., 1970, 1979).

$$
F_{\text {hyd }} \geq F_{\text {floc }}
$$

Thus, obtaining the strength of flocs against breakup is crucial for predicting transport of contaminants with colloidal particles in aquatic environments.

Direct measurement of floc strength was performed by Yeung and Pelton (1996), who measured the strength of flocs from the distortion of two micropipettes rupturing a floc. From the measurement, they found that floc strength does not change with floc size. This method gives direct information but to obtain strengths is time consuming. Other methods obtaining floc strength are the analysis of the maximum flocs size as a function of hydrodynamic disrupting force on flocs on the basis of the force balance given by Eq. (1) (Boller and Blaser, 1998; Kobayashi et al., 1999, 2002; Tambo et al., 1970, 1979). Blaser (2000) evaluated the hydrodynamic force acting on flocs and analyzed the 
motion of flocs in laminar flow fields by assuming that flocs behave as solid ellipsoids. He compared the results with observed motion of flocs in laminar flows and concluded that the motion of flocs is similar to those of solid ellipsoids. Blaser (2002) also calculated the hydrodynamic rupture force of fluid with a viscosity of $\mu$ exerted on ellipsoidal flocs with a surface area, $\mathrm{S}$, in an axisymmetric straining flow with an elongation rate, A. His results can be expressed by the following equation

$$
F_{\text {hyd }}=C_{\text {hyd }} S \mu A / 2 \text {. }
$$

In Eq. (2), $\mathrm{C}_{\text {hyd }}$ denotes the constant depending on floc shape and is the same as $\langle\mathrm{f}\rangle_{\mathrm{x}} / \mu \mathrm{A}$ in the paper (Blaser, 2002), where $\langle\mathrm{f}\rangle_{\mathrm{x}}$ is the hydrodynamic rupturing stress averaged over the half area of an ellipsoid (S/2). An axisymmetric straining flow is generated by the converging flow into an orifice. Studies on floc breakup by orifice converging flows were carried out by Sonntag and Russel (1987), Higashitani et al (1991), Blaser (2000a), and Kobayashi (2004). Kobayashi (2004) deduced that the maximum floc size subjected to the flow with a volumetric flow rate of Q through an orifice with a radius of $\mathrm{R}$ is determined by

$$
A_{c, \text { max }}=\frac{3 \sqrt{3} Q}{32 \pi R^{3}},
$$

which is the highest elongation rate of the flow along the centerline (Blaser, 2000a). This deduction is because flocs are subjected to higher stress when they flow along streamlines near wall (Sonntag and Russel, 1987). On the basis of this analysis, Kobayashi (2004) evaluated the strength of latex 
flocs using the following equation:

$$
F_{\text {floc }}=\left(C_{\text {hyd }} S\right)_{\text {max }} A_{c, \max } \mu / 2
$$

where $\left(\mathrm{C}_{\text {hyd }} \mathrm{S}\right)_{\max }$ denotes maximum values of $\mathrm{C}_{\text {hyd }} \mathrm{S}$ of flocs subjected to the flow with $\mathrm{A}_{c, \max }$, which is a function of Q and R. Evaluated strengths were reasonable when compared with the results by earlier studies; breakup of flocs with flocculants and coagulants in converging flows (Higashitani et al., 1991; Blaser, 2000a), breakup of flocs using micropipettes (Yeung and Pelton, 1996), pull-off force between latex particles with colloid-probe AFM (Hodges et al., 2002).

In the present study, the method to obtain floc strength described above was applied to flocs formed by the coagulation of natural soil colloidal particles, sampled from sugar cane field in Okinawa, Japan. The soil particles were coagulated in $0.5 \mathrm{M} \mathrm{NaCl}$ solution, $0.1 \mathrm{M} \mathrm{CaCl}_{2}$ solution or weakly acidified distilled water. The coagulated flocs were disrupted by a converging flow of the solutions. Experimentally obtained relationships between floc size and the elongation rate of the flow were analyzed by assuming flocs hydrodynamically behave like solid axisymmetrical elongated ellipsoids to evaluate strength of flocs with Eq. (4). Obtained strengths of soil flocs were $0.3 \mathrm{nN}, 0.7$ $\mathrm{nN}$ and $4 \mathrm{nN}$ for Na-coagulated flocs, Ca-coagulated flocs, and H-coagulated flocs, respectively. Furthermore, strengths of natural soil flocs did not depend on floc size. This finding is in agreement with previous studies on complex flocs (Yeung and Pelton, 1996) and simple latex flocs (Kobayashi, 2004). 


\section{Experimental Section}

Experiments were carried out to obtain the relationship between broken floc size and volumetric flow rate, which is related to elongation rate of converging flow disrupting flocs. Experimental methods described below are almost the same as the experiment previously performed for latex flocs (Kobayashi, 2004).

\subsection{Materials and floc formation}

Soil particles were sampled form the surface of the field of sugar cane in Onna-son, Okinawa-ken, Japan. In this region, soil erosion and resulting pollution of coastal area by suspended solids are regarded as serious environmental problems (Onaga, 1992; Nishimura and Mihara, 1993). To reduce the leakage of suspended solid into channel and river, many sediment basins were constructed. The soil is red-yellow colored and called Kunigami Maaji. Main mineral compositions of Kunigami Maaji are halloysite, illite, vermiulite, and goethite (Yang et al., 1998). The sampled soil particles were passed through a $74 \mu \mathrm{m}$ sieve and then immersed in $1 \mathrm{M} \mathrm{NaCl}$ solution more than 1 week to saturate particle surface with $\mathrm{NaCl}$. Then, the soil particles were purified by repeated sedimentation, discarding of supernatant and addition of distilled water until the conductivity of supernatant became below $10 \mu \mathrm{S} / \mathrm{cm}$, which corresponds to below $10^{-4} \mathrm{M} \mathrm{NaCl}$. Soil colloidal particles smaller than 5 
$\mu \mathrm{m}$ were collected by removing larger particles by sedimentation and were used for the present study.

The median diameter and the density of soil colloidal particles were $0.23 \mu \mathrm{m}$ and $2.79 \mathrm{~g} / \mathrm{cm}^{3}$. The soil particles were well coagulated in acidic solutions and dispersed in alkaline solutions.

Four types of flocs were formed by the coagulation of the soil colloidal particles with $0.5 \mathrm{M} \mathrm{NaCl}$ at $\mathrm{pH}$ 5.4-5.5, $0.5 \mathrm{M} \mathrm{NaCl}$ at $\mathrm{pH}$ 6.6, $0.1 \mathrm{M} \mathrm{CaCl}_{2}$ at $\mathrm{pH}$ 6.4-6.9, or the weakly acidified water $(\mathrm{pH}$ 5.6) made by the addition of a small amount of $0.1 \mathrm{M} \mathrm{HCl}$ in sample bottles. From now on, these flocs are called Na-coagulated, Ca-coagulated flocs, or H-coagulated flocs. For the coagulation at pH 6.4-6.9, $5 * 10^{-5} \mathrm{M} \mathrm{NaHCO}_{3}$ were added to keep the value of $\mathrm{pH}$ constant. These $0.5 \mathrm{M} \mathrm{NaCl}$ and 0.1 $\mathrm{M} \mathrm{CaCl}_{2}$ solutions were confirmed to be in the range of rapid coagulation by simple sedimentation experiments (Nishimura and Mihara, 1993). Flocs were fully coagulated, until they were settled.

\subsection{Converging flow generation}

Fig. 1 schematically illustrates experimental setup used in this study. A silicone stopper, in which a glass capillary tube with an inner diameter of $0.89 \mathrm{~mm}$ was inserted, was connected to one side of the larger glass tube with an inner diameter of $2.5 \mathrm{~cm}$ and a length of $22.5 \mathrm{~cm}$. A certain volume of electrolyte solution was poured into the larger tube. Then, sucking the solution from the larger tube into a capillary tube with a syringe pump through silicone tubing generated a converging flow at the vicinity of entrance of the capillary. The pump controls volumetric flow rates. Three volumetric flow 
rates, $0.17,0.33$, and $0.65 \mathrm{~mL} / \mathrm{s}$ were applied in this experiment. The Reynolds numbers of flows in the capillary were below the critical Reynolds number. Flocs moving toward the capillary were not subjected to turbulence. Thus, laminar flows are assumed in the present analysis.

\subsection{Procedure}

Sufficiently coagulated and settled flocs were transferred into the converging flow device by inserting one side of a glass tube with an inner diameter of $1 \mathrm{~cm}$ in the suspension containing sufficiently coagulated flocs in the bottle, trapping the suspension by capping another side of the tube with a finger, and gently pouring the suspension in $0.5 \mathrm{M} \mathrm{NaCl}$ solution, $0.1 \mathrm{M} \mathrm{CaCl}_{2}$ solution, or distilled water in the larger pipe of the flow device. Then, flocs were broken up by the converging flow into the capillary generated with the syringe pump at a prescribed volumetric flow rate. After breakup of flocs, the suspension containing disaggregated flocs in the silicone tubing were transferred into the O-ring with a depth of $3 \mathrm{~mm}$ connected to a glass slide by pushing gently the syringe with the pump. The suspension in the O-ring was covered with a thin slide glass and put on the stage of a digital microscope for capturing digital images of flocs. Lengths of major and minor axes ( $d_{\text {maj }}$ and $d_{\text {min }}$ ) of the best-fit ellipse corresponding to respective flocs were measured from the captured images by using the software Scion Image.

Two to Three experiments for each floc were conducted at room temperature $\left(24-25{ }^{\circ} \mathrm{C}\right)$. The 
viscosities of $\mathrm{NaCl}$ and $\mathrm{CaCl}_{2}$ solutions and water at $25{ }^{\circ} \mathrm{C}$ were used in the following analysis.

\section{Results and Discussion}

In Fig. 2, maximum values of $d_{\text {maj }}$ are plotted against flow rates, $Q$. As indicated in this graph, $\mathrm{H}$-coagulated flocs are largest among the present flocs and Ca-coagulated flocs are larger than Na-coagulated flocs. There is no clear effect of $\mathrm{pH}$ for Na-coagulated flocs in the present experimental conditions.

Floc strengths were obtained by assuming that flocs were elongated axisymmetric ellipsoids. Surface areas of best-fit ellipsoids corresponding to flocs, $\mathrm{S}$, were calculated by substituting $\mathrm{d}_{\text {maj }}$ and $d_{\min }$ of the fitted ellipses into the following equation (Moriguchi, 1987)

$$
S=2 \pi\left(a^{2}+\frac{a c^{2}}{\sqrt{c^{2}-a^{2}}} \arccos \frac{a}{c}\right)
$$

with $2 \mathrm{a}=\mathrm{d}_{\min }, 2 \mathrm{c}=\mathrm{d}_{\text {maj }}$. Then, $\mathrm{C}_{\text {hyd }}$ were obtained from the values listed in table 1 using the ratio of $\mathrm{d}_{\text {maj }} / \mathrm{d}_{\text {min. }}$. Using these values, maximum values $\left(\mathrm{C}_{\text {hyd }} S\right)_{\max }$ were determined for all experimental conditions. Finally, floc strengths were evaluated by Eq. (4) as discussed in introduction and the previous paper (Kobayashi, 2004).

Evaluated floc strengths are plotted against maximum major lengths, $d_{\text {maj,max }}$, in Fig. 3. In this graph, experimental data by previous researchers, direct rupturing of floc with micropipettes and floc breakup by converging flow, are also plotted. Details of these experimental conditions are given in 
Table 2. The mean values of floc strengths of natural soil colloids of this study were $0.3,0.7$, and 4 nN for Na-coagulated, Ca-coagulated, and H-coagulated flocs, respectively. Fig. 3 also indicates that strengths of natural soil flocs do not change with their size as found for breakup of latex flocs by flows (Kobayashi, 2004) and direct breakup by means of micropipettes (Yeung and Pelton, 1996).

From Fig. 3, one can find that floc strengths of this study were 1-3 orders of smaller than those of flocs formed with polymeric flocculants and/or precipitated coagulants (Al and Fe) (Blaser, 2000a; Higashitani et al., 1991; Yeung and Pelton, 1996). This conclusion is reasonable because flocculants and coagulants are used to increase strength of flocs in water and wastewater treatment. These low floc strengths mean that flocs are easily redispersed and cannot settle down. This effect is considered to be one of the reasons causing the problems; soil erosion and resulting pollution of coastal area.

Ca-coagulated flocs were found to have 2.5 times strength of Na-coagulated flocs even in rapid coagulation regime, where electrical repulsive forces can be negligible, according to the Derjaguin-Landau-Verwey-Overbeek theory of colloid stability. It is known that Ca ions inhibit the decrease of hydraulic conductivity of soil due to clogging induced by the dispersion of colloidal particles (Kretzschmar et al., 1999; Toride and Nishimura, 2003), restrict the swelling of montmorillonite sheets (Kjellander et al., 1990) and improve the efficiency of solid-liquid separation in water treatment (Sobeck and Higgins, 2002). To the author's knowledge, this study is the first to quantify the difference of strengths between Ca-coagulated and Na-coagulated soil flocs. Higher 
strengths of Ca-coagulated flocs are considered to be due to the bridging of surfaces by divalent ions

(Sobeck and Higgins, 2002) or strong attraction due to ion-ion correlation (Kjellander et al., 1990).

In addition, H-coagulated flocs were found to be about 10 times stronger than Na-coagulated flocs.

This effect is induced by several mechanisms, such as, dissolution and precipitation of aluminum or ferric ions sorbed on particle surfaces (Stumm and Morgan, 1996), or hetero-coagulation due to the attractive electrical double layer interaction between oppositely charged particle surfaces (Ryde and Matijevic, 1994; Yu and Borkovec, 2002). In the former case, flocs have the same strong strength as the flocs formed with precipitated coagulants used in water purification engineering. In the latter case, this effect might be important since the present soils, Kunigami maaji, contain goethites, which usually have positive charges in lower $\mathrm{pH}$ regimes (Hiemstra et al., 1989) and thus give rise to strong double layer attractive force especially in lower ionic strength (Ryde and Matijevic, 1994; Yu and Borkovec, 2002).

The reasons for the difference in strength between these flocs still remain as an unsolved problem.

To gain understanding of these effects, quantitative mechanical tests by the present method with chemically well-characterized particles are considered to be useful.

\section{Conclusions}

Floc strength can be obtained from a simple experiment of breakup of flocs subjected to a laminar 
converging flow into an orifice. In this study, this method was applied to natural soil flocs collected from the surface of sugar cane field in Okinawa Island, Japan. The soil flocs were coagulated with 0.5 M NaCl (pH 5.4-5.5 or $\mathrm{pH}$ 6.6) solutions, $0.1 \mathrm{M} \mathrm{CaCl}_{2}$ (pH 6.4-6.9) solutions, which are above the critical coagulation concentrations, or acidified distilled water with dilute $\mathrm{HCl}(\mathrm{pH}$ 5.6). Obtained floc strengths were $0.3 \mathrm{nN}, 0.7 \mathrm{nN}$ and $4 \mathrm{nN}$ for Na-coagulated flocs, Ca-coagulated flocs, and H-coagulated flocs, respectively. Furthermore, floc strength did not change with floc size. These values of floc strengths were 1-3 orders smaller than those of flocs formed by the addition of polymer flocculants and/or precipitated ferric or aluminum hydroxide coagulants generally used in water treatments.

\section{Acknowledgments}

The author is thankful to Dr Yasuhisa Adachi at University of Tsukuba and Dr Yuji Maejima at University of Tokyo for their fruitful discussion and to Prof. Michal Borkovec at University of Geneva and Prof. Tadatsugu Tanaka at University of Tokyo for their guidance throughout this study. Japan Society for the Promotion of Science financially supports the author (2000-2003, 2003-2005). 


\section{References}

Blaser, S. (2000a) Break-up of flocs in contraction and swirling flows. Colloids and Surfaces A 166, $215-223$

Blaser, S. (2000b) Flocs in shear and strain flows. J. Colloid Interface Sci., 225, 273-284

Blaser, S. (2002) Forces on the surfaces of small ellipsoidal particles immersed in a linear flow field. Chem. Eng. Sci., 57, 515-526

Boller, M. and Blaser, S. (1998) Particles under stress. Water Sci. Tech., 37, 9-29

Droppo I. G. (2001) Rethinking what constitutes suspended sediment. Hydrol. Process. 15, $1551-1564$

Edzwald J. K., Upchurch J. B. and O’Melia C. R. (1974) Coagulation in estuaries. Environ. Sci. Tech. 8, 58-63

Hiemstra, T., De Wit, J. C. M., and Van Riemsdijk, W. H. (1989) Multisite proton adsorption modeling at the solid/solution interface of (hydr)oxide: A new approach II. Application to various important (hydr)oxide . J. Colloid Interface Sci., 133, 105-117.

Higashitani, K., Inada, N., and Ochi, T. (1991) Floc brekup along centerline of contractile flow to orifice. Colloids and Surfaces 56, 13-23

Hodges, C. S., Cleaver, J. A. S., Gahdiri, M, Jones, R., and Pollock, H. M. (2002) Forces between polystyrene in water using the AFM: Pull-off force vs particle size. Langmuir, 18, 5741-5748 
Kjellander R., Marcelja S., Pashley R. M. And Quirk J. P. (1990) A theoretical and experimental study of forces between charged mica surfaces in aqueous $\mathrm{CaCl}_{2}$ solutions. J. Chem. Phys. 92, 4399-4407

Kobayashi, M., Adachi, Y. and Ooi, S. (1999) Breakup of fractal flocs in a turbulent flow. Langmuir, 15, 4351-4356

Kobayashi, M., Adachi, Y., and Ooi, S. (2002) The effects of particle size on breakup of polystyrene latex flocs in a turbulent flow. J. Hydroscience and Hydraulic Engineering, 20, 179-186

Kobayashi, M. (2004) Breakup and strength of polystyrene latex flocs subjected to a converging flow, Colloids and Surfaces A, 235, 73-78.

Kretzschmar R., Brokovec M., Grolimund D. and Elimelech M. (1999) Mobile subsurface colloids and their role in contaminant transport. Adv. Agronomy, 66, 121-193

Moriguchi S. (1987) Mathematical formulas (Sugaku Koshikishu), Iwanami Shoten, Tokyo, in Japanese

Nishimura T. and Mihara M. (2003) Soil erosion and transport of colloids, in Soil Colloidal Phenomena (Tsuchi no Colloid Gensho), Y. Adachi and S. Iwata Eds., Gakkai Shuppan Center, Tokyo, 267-276, in Japanese

Onaga K. (1992) The present state of soil conservation in Japan. Australian J. Soil and Water Conservation, 5, 41-45 
Ryde N. and Matijevic E. (1994) Kinetics of heterocoagulation part 4. Evaluation of absolute coagulation rate constant using a classical light scattering technique, J. Chem. Soc. Faraday Trans., 90, $167-171$

Sobeck, D. C. and Higgins, M. J. (2002) Examination of three theories for mechanisms of cation-induces bioflocculation. Water Res., 36, 527-538

Sonntag, R. C. and Russel, W. B. (1987) Structure and breakup of flocs subjected to fluid stresses: III. Converging flow. J. Colloid Interface Sci., 115, 390-395.

Stumm, W. and Morgan, J. J. (1996) Aquatic Chemistry, John Wiley \& Sons, New York

Tambo N. and Hozumi H. (1979) Physical characteristic of flocs. 2. Strength of floc. Water Res., 13, 421-427

Tambo, N., Yamada, K. and Hozumi, H. (1970) Study on floc strength(Furokku kyodo ni kansuru kenkyu), Suido Kyokai Zasshi, 427 4, in Japanese

Toride N. and Nishimura T. (2003) Colloid stability and hydraulic conductivity of Soil, Soil Colloidal Phenomena (Tsuchi no Colloid Gensho), Adachi Y. and Iwata S. Eds., Gakkai Shuppan Center, Tokyo, 276-291, in Japanese

Yang J., Onaga K., Gibo S. and Tokashiki Y. (1998) Erosion control of Kunigami-maaji soil by applying of different organic matters. Trans. JSIDRE (Nogyo Doboku Gakkai Ronbunshu), 198, 161-168, in Japanese with English summary 
Yeung, A. K. C. and Pelton, R. (1996) Micromechanics: A new approach to studying the strength and breakup of flocs, J. Colloid Interface Sci., 184, 579-585

Yu W. L. and Borkovec M. (2002) Distinguish heteroaggregation from homoaggregation in mixed binary particle suspensions by multiangle static and dynamic light scattering. J. Phys. Chem. B, 106, $13106-13110$ 


\section{Figure captions}

Figure 1 Schematic illustration of experimental setup.

Figure 2 Maximum broken floc size against volumetric flow rate of flow.

Figure 3 Floc strength against floc size. 
Table $1 \mathrm{Chyd}_{\text {hyd }}$ for the evaluation of hydrodynamic force on a floc. These values are extracted from the contour graph in the paper by Blaser(2002).

\begin{tabular}{cc}
\hline $\mathrm{d}_{\text {maj }} / \mathrm{d}_{\text {min }}$ & $\mathrm{C}_{\text {hyd }}$ \\
\hline $1-1.6$ & 5 \\
$1.6-2.8$ & 5.25 \\
& \\
$2.8-3.6$ & 5.5 \\
& \\
$3.6-4.2$ & 5.75 \\
\hline
\end{tabular}


Table 2 Experimental conditions of breakup studies cited in Fig. 3.

\begin{tabular}{|c|c|c|c|}
\hline Authors & Colloidal particles & $\begin{array}{l}\text { Flocculants } \\
\text { or coagulants }\end{array}$ & $\begin{array}{l}\text { Methods of floc breakup } \\
\text { and representative floc size } \\
\text { (X-axis in Fig 3) }\end{array}$ \\
\hline This study & $\begin{array}{c}\text { Natural soil colloidal } \\
\text { particles }\end{array}$ & $\begin{array}{c}0.5 \mathrm{M} \mathrm{NaCl} \\
0.1 \mathrm{M} \mathrm{CaCl}_{2} \\
\text { No salt (acidic with dilute } \mathrm{HCl} \text { ) }\end{array}$ & $\begin{array}{l}\text { Converging flow. } \\
\text { Maximum major length of } \\
\text { best-fit ellipse. }\end{array}$ \\
\hline Blaser & $\begin{array}{l}\text { Latex spheres } \\
\text { (styrene/acrylate) with a } \\
\text { diameter of } 140 \mathrm{~nm} \text {. }\end{array}$ & $\begin{array}{l}1.9 * 10^{-5} \mathrm{M} \mathrm{FeCl}_{3} 6 \mathrm{H}_{2} \mathrm{O}(5 \mathrm{mg} / \mathrm{L} \\
\mathrm{Fe}) \text { in mineral water (ionic strength } \\
\text { of } 0.01 \mathrm{M}, \mathrm{pH} 7)\end{array}$ & $\begin{array}{l}\text { Converging flow. } \\
\text { Equivalent sphere area } \\
\text { diameter of each floc. }\end{array}$ \\
\hline Yeung \& Pelton & $\begin{array}{c}\text { Precipitated calcium } \\
\text { carbonate with an average } \\
\text { diameter of } 1.4 \mu \mathrm{m} .\end{array}$ & $\begin{array}{l}\text { A flocs: nonionic block copolymer } \\
\text { (polyacrylamide backbone with } \\
\text { pendant PEG chains) and } \\
\text { copolymer of vinylphenol and } \\
\text { sodium p-styrene sulfate. } \\
\text { B flocs: a cationic copolymer of } \\
\text { acrylamide and a bentonite clay. }\end{array}$ & $\begin{array}{c}\text { Two micropipettes. } \\
\text { Feret (perpendicular to } \\
\text { pulling direction) diameter } \\
\text { of each floc. }\end{array}$ \\
\hline $\begin{array}{c}\text { Higashitani et } \\
\text { al }\end{array}$ & $\begin{array}{c}\text { Latex spheres } \\
\text { (styrene/divinilbenzene) with } \\
\text { a diameter of } 90 \mu \mathrm{m} \text {. }\end{array}$ & $\begin{array}{l}\text { Glycerol solution of } 80 \mathrm{~g} / \mathrm{m}^{3} \\
\text { aluminum sulfate and } 20 \mathrm{~g} / \mathrm{m}^{3} \\
\text { polyacrylamide. }\end{array}$ & $\begin{array}{l}\text { Converging flow (breakup } \\
\text { of doublets). } \\
\text { Primary particle diameter } \\
\qquad(90 \mu \mathrm{m}) .\end{array}$ \\
\hline
\end{tabular}




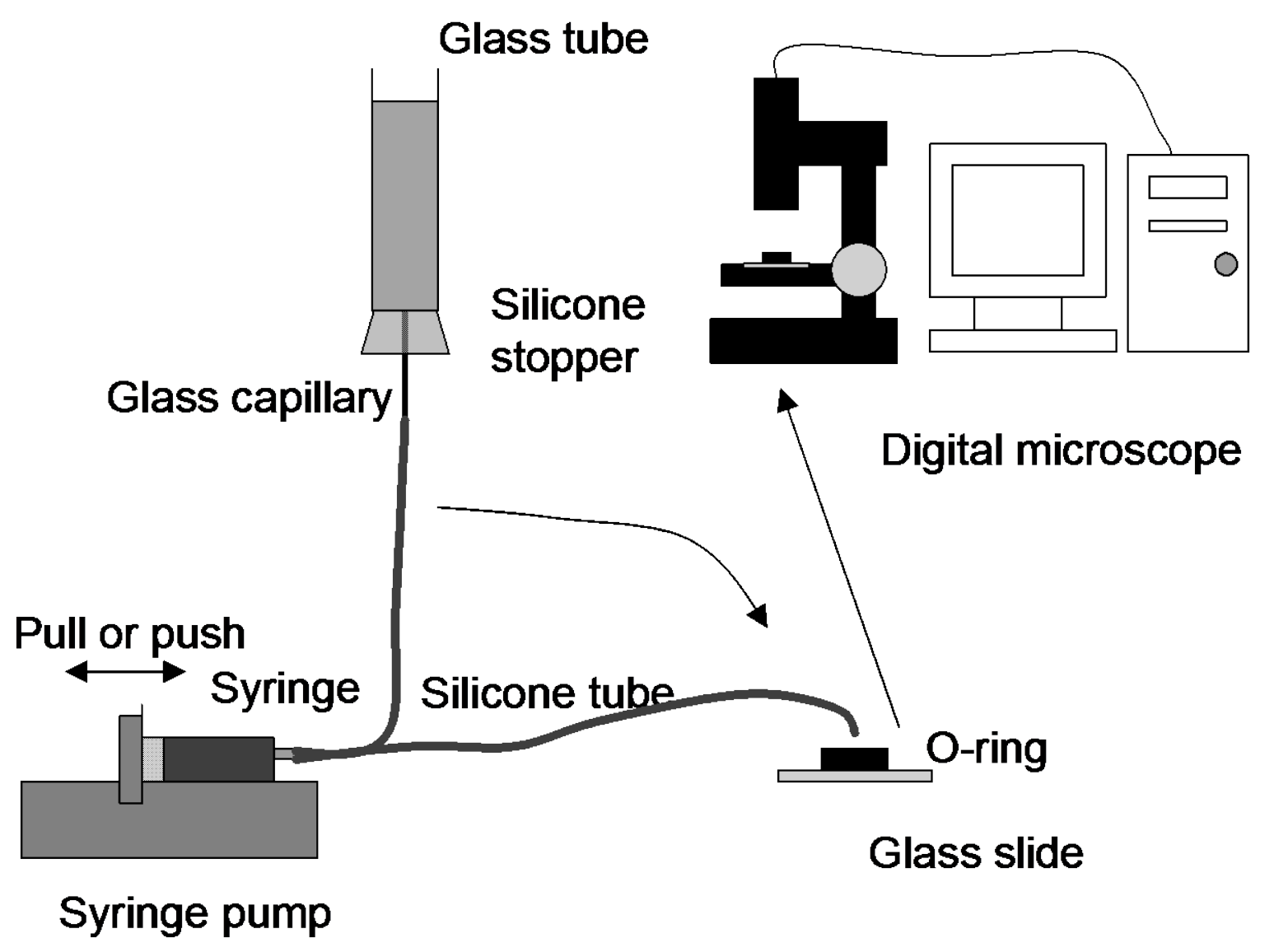

Fig 1 


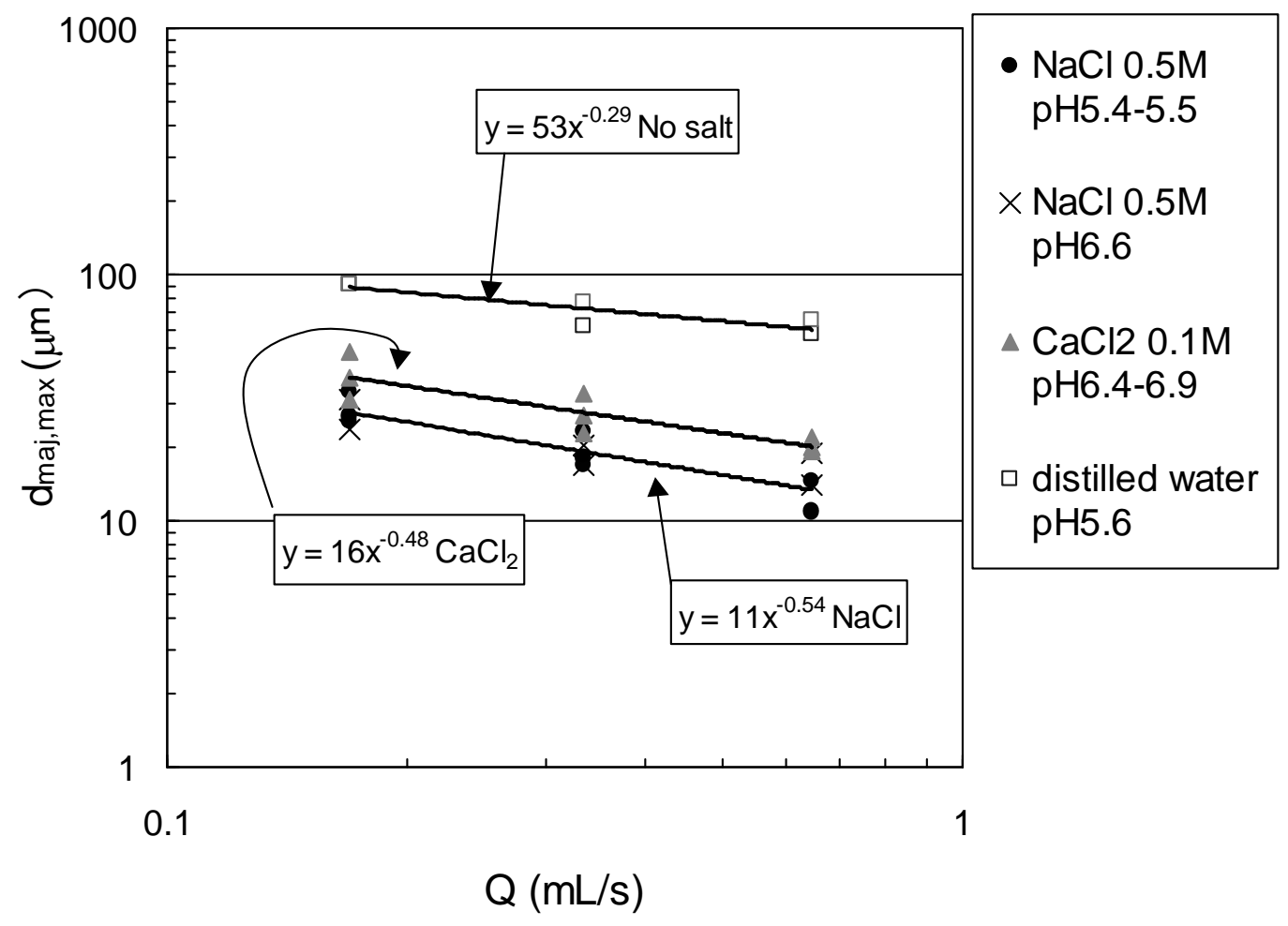

Fig. 2 


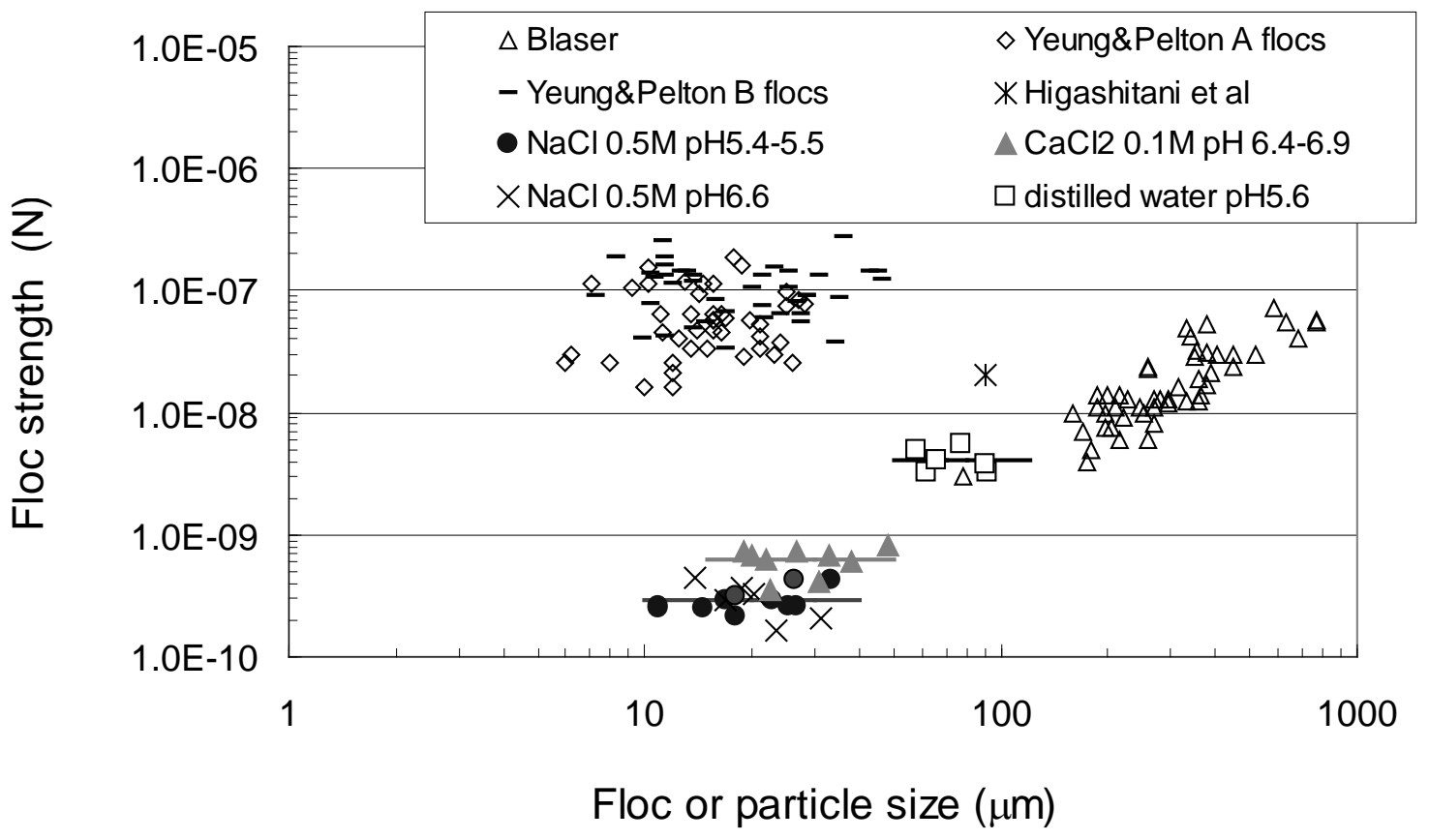

Fig. 3 